\title{
Perfect Anti-windup in Output Tracking Scheme with Preaction
}

\author{
$\begin{array}{lll}\text { P. Mercorelli } & \text { B. Paden } & \text { D. Prattichizzo† }\end{array}$ \\ tDipartimento di Ingegneria dell'Informazione, Università di Siena \\ via Roma, 56, 53100 Siena, Italy \\ mercorel(prattichizzo)@ing.unisi.it \\ $\ddagger$ Department of Electrical Engineering and Conputer Science, University of California at S. Barbara \\ 93106 Santa Barbara, California USA \\ paden@engineering.ucsb.edu
}

\begin{abstract}
This paper proposes a dynamic anti-windup (AW) compensation scheme for linear control systems with saturating actuators. Perfect tracking is achieved using preactuation and a dynamic compensator controlling the reset windup phenomenon. Two performance indices are considered and an example is included to illustrate the effectiveness of the proposed method.
\end{abstract}

\section{Introduction and Motivation}

Industrial processes impose nonlinear limits on their process variables, while linear design techniques assume that there are no such limits. All physical systems need actuators for achieving control and these are subject to saturation. When this happens the feedback loop is effectively broken and if a controller with an integrator is used the error will continue to be integrated. The value at the output of the controller can become very large and often degrades the closed-loop performance in the form of large overshoot, long settling time and sometimes even instability. The phenomenon described is called "windup".

In [7] the importance and the effectiveness of the preaction for solving the output tracking problem in nonminimum phase systems is emphasized. The importance of the preview control is well known in industrial applications, where it is often possible to know the reference signal in advance. The perfect output tracking is an ambitious objective for every system and recently, since the end of the sixties, has been pointed out in connection with optimal tracking. More recently perfect output tracking has been proposed for nonlinear systems in [5] and in [7] for linear systems.

When these ideas are applied in practical systems real actuators degrade the theoretically predicted performance. This means that the efforts for achieving per- fect tracking could be wasted in the presence of saturating actuators if an adequate anti-windup scheme is not used. This paper suggests a way to resolve this problem in the case of linear time invariant (LTI) systems, and at the same time proposes a complete scheme (fig. 3) for reducing deterioration caused by windup phenomenon. The anti-windup scheme is independent from the preaction but obviously the feedforward control improves performances. The approach is similar to other formulation of the anti-windup problem in control systems $[4,9,10]$. It consists of recognizing the presence of windup and of finding a feedback compensator able to reset the integrator to a 'normal' value.

\section{Perfect Tracking Using Preaction}

The control philosophy illustrated in fig. 1 is based on a feedforward compensation scheme which was recently presented in $[7,6]$. The controller $C(s)$ is completely standard and may include the internal model of an exosystem reproducing a particular reference input. The feedforward compensator $K(s)$ generates an input correction that tends to reduce the magnitude of $u_{1}(t)$ (fig. 1). Finally the filter $f(s)$ is chosen such that $f(s) P^{-1}(s)$ is biproper. Note that filter $f(s)$ must be diagonal in order to not introduce any change in the output direction.

If the plant is minimum phase the structure of the compensator $K(s)$ is set equal to $f(s) P^{-1}(s)$. If the plant is non-minimum phase the compensator $f(s) P^{-1}(s)$ would be unstable, thus it is not feasible and the structure of the feedforward compensator should be more involved. In particular in $[7,6]$ it was proved that the effect of the unstable component is equivalent to a free response of the plant and can be regarded as a initial condition. Thus it will be sufficient to take the plant to this initial condition with a preaction signal which maintains the output identically zero. For achieving this it is necessary to know the reference signals with

\subsection{3-4104-X/98/\$10.00 C1998 IEEE}




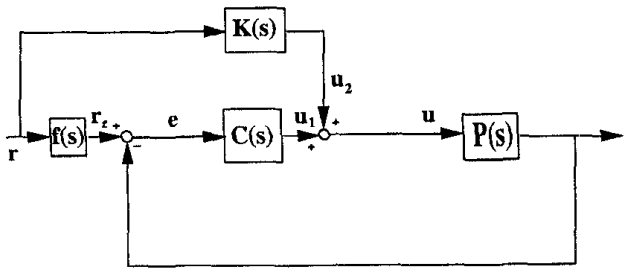

Figure 1: A control system with preaction, without saturation.

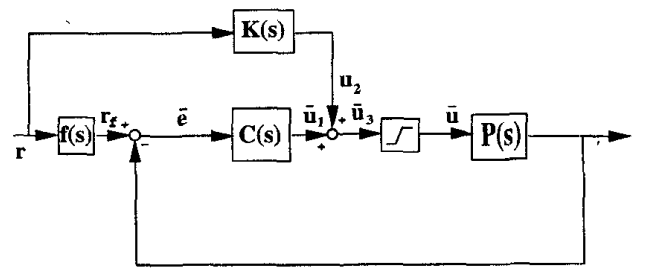

Figure 2: A control system with preaction and saturating actuator.

for an infinite time in advance. Fortunately it is always possible to approximate that initial condition, the precision of the approximation depends on the preaction time $t_{\text {pre }}$ and will be improved for larger $t_{\text {pre }}$. Moreover, in [7] it was proved that with a special choice of $K(s)$ it holds

a) $\|e(t)\| \rightarrow 0$ as $t_{\text {pre }} \rightarrow-\infty$

b) $\int_{-\infty}^{\infty}\|e(t)\|^{2} \rightarrow 0$ as $t_{p r e} \rightarrow-\infty$.

\section{Perfect Anti-Windup}

Consider the scheme in fig. 2 and assume that, except for saturating actuators, the system is LTI. According to techniques for designing $K(s)$ based on preaction $[7,6]$, the perfect tracking or almost perfect tracking is achieved also for non minimum phase plants. Unfortunately such those techniques work only without saturation. When saturation occurs, perfect tracking is lost and corrective design action is needed. The method presented in this paper consists of recognizing the windup phenomenon and then adjusting error $\bar{e}(t)$ so that the controller $C(s)$ is not affected by saturation. For achieving the goal it will be enough to feed back a dynamic controller $N(s)$ in the conventional anti-windup (CAW) scheme of fig. 3 .

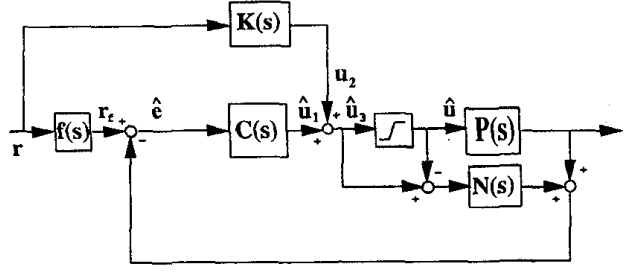

Figure 3: A control system with preaction, saturating actuator and anti-windup scheme.

\subsection{Problem Formulation}

The classical anti-windup schemes, normally developed for PI or PID controllers, involve turning off the integral action in the presence of saturation. This paper is focused on the control system with preaction, saturating actuator and anti-windup presented in fig. 3 . Windup can be interpreted as an inconsistency of the output or the state of the controller between the case with (fig. 1) and the case without saturation (fig. 3) $[10,9]$. Differences between the satured and unsatured signals can be quantified by these two cost functions

$$
J_{1}=\int_{0}^{\infty}\left\|u_{1}(t)-\hat{u}_{1}(t)\right\|^{2} d t
$$

and

$$
J_{2}=\int_{0}^{\infty}\left\|x_{c}(t)-\hat{x}_{c}(t)\right\|^{2} d t
$$

where $\|\cdot\|$ represents the Euclidean norm, and $u_{1}(t)$ and $x_{c}(t)\left(\hat{\mathbf{u}}_{1}(t)\right.$ and $\left.\hat{x}_{c}(t)\right)$ are the output and the state, respectively, of the controller in the scheme without (with) saturating actuators.

In what follows the problem of minimizing the cost functions $J_{1}$ and $J_{2}$ over the feasible controller $N(s)$ is investigated.

\subsection{Feedback Compensator}

This section deals with the cost function weighting the output of controller $C(s)$. A few assumptions are necessary for solving the minimization problem the compensator $N(s)$ :

A1) The plant $P(s)$ is stable, strictly proper and without zeros on imaginary axis.

A2) The closed-loop System of fig. 1 is stable.

A3) The initial condition of the plant is zero.

Proposition 1 Consider the scheme shown in fig. 3. Under assumptions $A 1-A 3$, the global minimum $J_{1}=$ 
0 of the performance index in eq. (1) is obtained for $N(s)=P(s)$.

Proof: The proof is straightforward. It starts from re-writing the performance index in the Laplace domain according to the Parseval Theorem.

\section{State Space Analysis}

This section is devoted to the the minimization of in$\operatorname{dex}(2)$ in the state space. Let the four-map and the three-map systems $(F, G, H, L)$ and $(A, B, C)$ represent minimal realizations of controller $C(s)$ and plant $P(s)$, respectively. Moreover, let $\mathcal{F}(t), \mathcal{K}(t)$ and $\mathcal{N}(t)$ be the impulse responses of the filter $f(s)$, the preaction $K(s)$ and the compensator $N(s)$, respectively.

With reference to fig. 3 , the dynamics of the plant is

$$
\begin{aligned}
& \dot{\hat{x}}_{p}(t)=A \hat{x}_{p}+B \hat{u}(t) \\
& \hat{y}(t)=C \hat{x}_{p} \\
& \hat{u}(t)=\operatorname{sat}\left(\hat{u}_{3}(t)\right)
\end{aligned}
$$

and the dynamics of the compensated controller [8]

$$
\begin{aligned}
& \dot{\hat{x}}_{c}(t)=F \hat{x}_{c}+G\left[\hat{e}(t)+\mathcal{N}(t) *\left(\hat{u}(t)-\hat{u}_{3}(t)\right)\right] \\
& \hat{u}_{3}(t)=H \hat{x}_{c}+L \hat{e}(t)+\mathcal{K}(t) * r(t) \\
& \hat{e}(t)=\mathcal{F}(t) * r(t)-\hat{y}(t)
\end{aligned}
$$

Next proposition shows how to minimize index $J_{2}(2)$.

Proposition 2 Under the hypothesis A1-A3 the dynamic compensator $N(s)=P(s)$, which minimizes $J_{1}$ (cf. Proposition 1), minimizes also the performance in$\operatorname{dex} J_{2}$ in eq. (2) if and only if $L=0$. The minimum obtained is global, $J_{2}=0$.

Proof: By Parseval Theorem, the performance index (2) can be rewritten as

$$
J_{2}=\frac{1}{2 \pi j} \int_{-j \infty}^{j \infty}\left\|X_{c}(s)-\hat{X}_{c}(s)\right\|^{2} d s .
$$

From the (3) and (4) the state of $C(s)$ in the control scheme of fig. 3 is

$$
\begin{aligned}
& \dot{\hat{x}}_{c}(t)=F \hat{x}_{c}(t)+G\left[\mathcal{F}(t) * r(t)-C \hat{x}_{p}(t)+\mathcal{N}(t) *\right. \\
& \left.\left[\hat{u}(t)-\left(H \hat{x}_{c}(t)+L\left(\mathcal{F}(t) * r(t)-C \hat{x}_{p}(t)\right)+\mathcal{K}(t) * r(t)\right)\right]\right]
\end{aligned}
$$

and its Laplace transform $(N(s)=P(s))$

$$
\begin{aligned}
\hat{X}_{c}(s) & =(s I-F+G P(s) H)^{-1}(G[f(s)-P(s) L f(s)- \\
& P(s) K] R(s)+G[P(s) L P(s)] \hat{U}(s))
\end{aligned}
$$

As regards the state of $C(s)$ in the scheme without saturation of fig. 1 , we obtain

$$
\begin{aligned}
& X_{c}(s)=\left(s I-F+G C(s I-A+B L C)^{-1} B H\right)^{-1} \\
& \quad G\left[f(s)-G C(s I-A+B L C)^{-1}(B L f(s)+K(s))\right] R(s) .
\end{aligned}
$$

The sufficiency is trivial. The necessary part of the proof follows by independence of $R(s)$ and $\hat{U}(s)$. Since $X_{c}$ depends only on $R(s), X_{c}=\hat{X}_{c}$ only if the coefficient of $\hat{U}(s)$ in (6) vanishes, i.e. $L=0$.

$\square$

Concerning the meaning for $L=0$ we can observe that our control system does a correction on the input of the regulator $C(s)$ as in $\mathrm{CAW}$ and it does not correct directly the state as in [9].

Remark 1 Note that the proof of Proposition 2 assumes that matrix $(s I-F+G P(s) H)$ is invertible. This is simply verified by observing that for $L=0$ the matrix of the closed loop system is

$$
A_{n s a t}=\left[\begin{array}{cc}
F & -G C \\
B H & A
\end{array}\right] .
$$

and its determinant, cf. [2]

$$
\begin{aligned}
& \operatorname{det}\left(s I-A_{n s a t}\right) \\
& \quad=\operatorname{det}(s I-A) \operatorname{det}\left[s I-F+G C(s I-A)^{-1} B H\right] .
\end{aligned}
$$

Normally the controller provides an acceptable nominal performance in the absence of saturating actuators and the condition $\operatorname{det}\left(s I-A_{n s a t}\right) \neq 0 \forall R e[s] \geq 0$ is easily achieved. Consequently $\operatorname{det}(s I-F+G P(s) H) \neq 0$ $\forall \operatorname{Re}[s] \geq 0$.

Remark 2 When we have saturating actuators it is not possible to have

a) $\|e(t)\| \rightarrow 0$ as $t_{\text {pre }} \rightarrow-\infty$

b) $\int_{-\infty}^{\infty}\|e(t)\|^{2} \rightarrow 0$ as $t_{\text {pre }} \rightarrow-\infty$

as in Theorem 4.1 of [7]. In fact in our case the state of the plant during the preaction is $\left\|x_{p}(t)\right\| \leq \delta$ and not $\left\|x_{p}(t)\right\| \leq \delta_{1} \exp ^{t_{p r e}}$ as stated in [7]. Because of saturation we have a fixed level of the final state during preaction. Thus according to the [7] we can write

$$
\|e(t)\| \leq \delta_{2} \exp ^{\sigma\left(t+t_{\text {pre }}\right)} \delta
$$


where $\sigma(<0)$ is the maximal real part of the eigenvalues of the dynamic matrices of system with and without saturation and where $\delta, \delta_{1}$ and $\delta_{2}$ are constants. For relationship a) it holds

$$
\|e(t)\| \leq \delta_{2} \delta
$$

and analogously for the index $b$ ). This means that while in case without saturating actuators we have the error tending to zero when $t_{\text {pre }} \rightarrow-\infty$ in presence of saturating actuators it is not possible to reduce to zero the error increasing the time of preaction. In fig. 7 and in fig. 8 it is shown an example where the error does not vanish.

Remark 3 In presence of a rate limiter rather than a simple saturation, an interesting property obtained using the preaction scheme is achieved, i.e. the reduction of the error tracking.

In fact if the speed magnitude of the post-action signal, see [6], is not greater than the rate limiter level, then it is possible to reduce to zero the tracking error when $t_{p} \rightarrow-\infty$. In fact it is easy to show that the speed magnitude of the preaction signal can be reduced to zero by increasing the preaction time $\left(t_{p} \rightarrow-\infty\right)$. The time derivative of the preaction signal for continuous systems can be written as

$$
v=\sum_{h=1}^{m} K_{h} Z_{h} \exp ^{Z_{h} t_{p}} \exp ^{Z_{h} t}
$$

where the $Z_{h}$ 's are the unstable zeros of the plant and $K_{h}$ 's are suitable weights [6].

Finally as regards stability, it should be remarked that normally it is a serious problem when the controller by itself is not stable, but not in this case where the compensator provides a perfect 'turn off' of the controller. It is easy to show that the system depicted in fig. 3 is BIBO stable directly from the fact that the plant is stable, the closed loop system is stable, the $\operatorname{sat}\left(u_{3}(t)\right)$ is a bounded function and $N(s)$ provides limitations on the output of the controller even if this were unstable.

\section{Example}

In this section one example is shown to demonstrate the effectiveness of the proposed method depicted in fig. 3 .

Example: Consider the following plant

$$
P(s)=-3 \frac{(s-2)(s+4)}{(s+1)(s+2)(s+8)}
$$

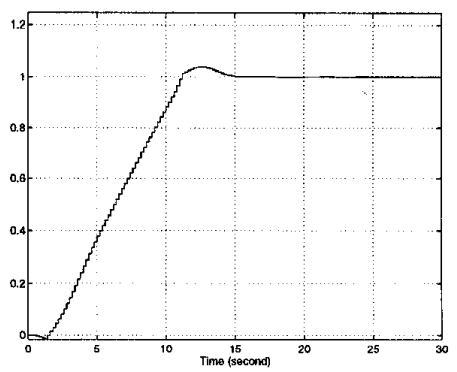

Figure 4: Output of the closed loop system without saturating actuator with $t_{\text {pre }}=5 \mathrm{sec}$.

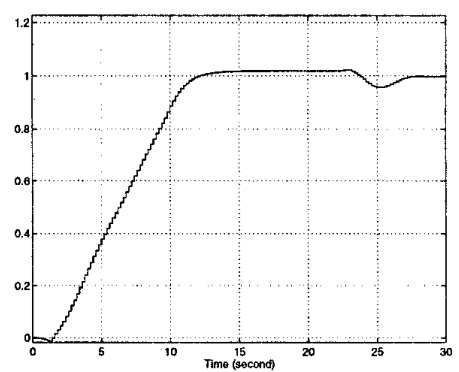

Figure 5: Output of the closed loop system with saturating actuator and without anti-windup compensator with $t_{\text {pre }}=5 \mathrm{sec}$.

with a sampling time $T=0.2$ sec. we achieve

$$
P(z)=-0.2452 \frac{(z-0.454)(z-1.534)}{(z-0.2019)(z-0.6703)(z-0.8187)}
$$

we can take a controller as

$$
C(z)=\frac{(z-0.9)^{2}+0.05^{2}}{(z-1)^{2}}
$$

The feedforward action is selected according to [6]. The input is set to a ramp of speed $0.1 / \mathrm{sec}$ saturated at amplitude equal to 1 , the saturation level of the actuator is equal to $|0.68|$ and the preaction time is set to $t_{\text {pre }}=5$ sec. Fig. 4 depicts the performance of the closed loop system without saturating actuator. Fig. 5 depicts the performance of the closed loop system with saturation of actuator but without anti-windup compensator. Fig. 6 depicts the performance of the closed loop system with actuator saturation and with anti-windup compensator. It is easy to see how the introduction of the saturating actuator deteriorates significantly the performance (large overshoot and long settling time) if compared with expected linear performance. 


\section{Conclusions}

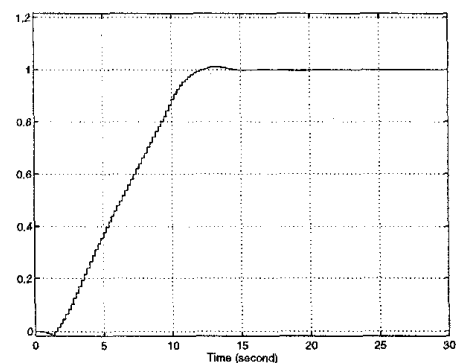

Figure 6: Output of the closed loop system with saturating actuator and anti-windup compensator with $t_{\text {pre }}=5$ sec.

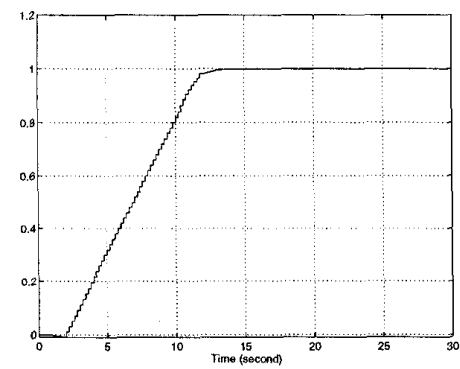

Figure 7: Output of the closed loop system with saturating actuator and anti-windup compensator with $t_{\text {pre }}=8 \mathrm{sec}$.

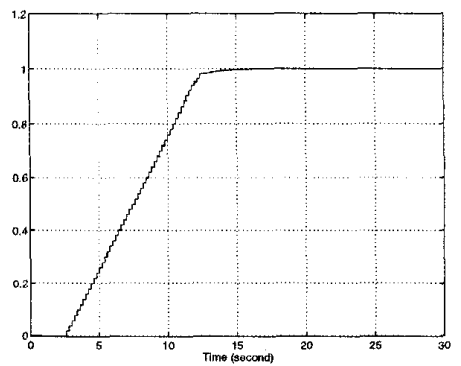

Figure 8: Output of the closed loop system with saturating actuator and anti-windup compensator with $t_{\text {pre }}=15 \mathrm{sec}$.
A dynamic anti-windup compensation method is proposed. In particular we considered the scheme that guarantees the perfect tracking using preaction because in this case the windup phenomenon could destroy completely the results achieved. The anti-windup compensator is based on the model of the controlled plant.

\section{Acknowledgements}

We would like to thank the people who contributed to this paper with ideas and discussions, particularly Maria Marta Seron and Julio Braslavsky of the Dep. Elect. Eng. and Computer Science (UCSB).

\section{References}

[1] K.J. Aström and L. Rundqwist, "Integrator Windup and How to Avoid It," in Proceeding of the ACC' 87 Pittsburgh.

[2] T. Kailath, Linear Systems. Englewood Cliffs, NJ:Prentice-Hall, 1980.

(3) N.Kapoor, A.R. Teel and P. Daoutidis, "An Invariant Subspace Technique for Anti-windup Synthesis," in Proceeding of the ACC '97 Albuquerque, New Messico, USA.

[4] M.V. Kothare, P.J. Campo, M. Morari and C.N. Nett, "A Unified Framework for the Study of AntiWindup Designs," in Automatica, 30:1869-1883,1994.

[5] S. Devasia, D. Chen and B. Paden, "Nonlinear Inversion-Based Output Tracking", in IEEE Transactions on Automatic Control, Vol. 41, NO. 7, July 1996.

[6] G. Marro and L. Marconi, "Using the Diophantine Equation in the Design of a Digital Perfect Tracking Compensator," in Proceeding of the ECC '97 Bruxelles $B E$.

[7] G. Marro and A. Piazzi, "A Geometric Approach to Multivariable Perfect Tracking, " in Proceeding of the IFAC' 96 San Francisco, CA, USA.

[8] P. Mercorelli, "Output Perfect Tracking scheme with anti-windup," Internal Report 4/98 Dipartimento di Ingegneria dell'Informazione, Università degli Studi di Siena, Italy.

[9] J. K. Park and C.-H Choi, "Dynamic compensation method for multivariable control systems with saturating actuator," in IEEE Trans. Aut. Contr., 40:1635-1640, 1995.

[10] A. Zheng, Mayuresh V. Kothare and M. Morari, "Anti-windup design for internal model control," in INT.J.Control, 1994 vol. 60, N. 5, 1015-1024. 